\title{
Antipsychotic Drugs Reverse MK801-Inhibited Cell Migration and F-actin Condensation by Modulating the Rho Signaling Pathway in B35 Cells
}

\author{
Yi-Chyan Chen $\left(\mathbb{D},{ }^{1}\right.$ Fu-Ming Tsai $\mathbb{D}^{2},{ }^{2,3}$ and Mao-Liang Chen $\mathbb{D}^{2}$ \\ ${ }^{1}$ Department of Psychiatry, Taipei Tzu Chi Hospital, Buddhist Tzu Chi Medical Foundation, New Taipei City, Taiwan \\ ${ }^{2}$ Department of Research, Taipei Tzu Chi Hospital, Buddhist Tzu Chi Medical Foundation, New Taipei City, Taiwan \\ ${ }^{3}$ Department of Microbiology, Soochow University, Shih Lin, Taipei City, Taiwan \\ Correspondence should be addressed to Mao-Liang Chen; cater0656@hotmail.com
}

Received 7 July 2020; Revised 13 October 2020; Accepted 29 October 2020; Published 11 November 2020

Academic Editor: Marco Carotenuto

Copyright (@) 2020 Yi-Chyan Chen et al. This is an open access article distributed under the Creative Commons Attribution License, which permits unrestricted use, distribution, and reproduction in any medium, provided the original work is properly cited.

\begin{abstract}
Background and Aim. MK801-induced psychotic symptoms and also the Ras homolog family member A (RhoA) expression and cell division control protein 42 ( $c d c 42)$ mRNA modulation in the rat brain have been investigated. Antipsychotic drugs (APDs) have been reported to induce Rho GDP-dissociation inhibitor (RhoGDI) pathway regulation related to cytoskeleton reorganization in neuronal cells. It will be necessary to clarify the effects of APDs on MK801-induced RhoGDI signaling regulation in neuronal cells. Methods. B35 neuronal cells were treated with MK801 for 7 days then treated with MK801 in combination with haloperidol or clozapine for a further 7 days. Cell migration, F-actin condensation, and RhoGDI signaling regulation were examined to investigate the regulatory effects of MK801, haloperidol, and clozapine in B35 neuronal cells. Results. MK801 reduced B35 cell migration, whereas both haloperidol and clozapine reversed the reduction in cell migration induced by MK801. Haloperidol and clozapine restored F-actin condensation after it was diminished by MK801 in B35 cell nuclei. MK801 increased the RhoGDI1 and RhoA expression, which was diminished by the addition of haloperidol and clozapine. MK801 reduced the CDC42 expression, which was restored by haloperidol and clozapine. MK801 reduced the Rhoassociated coiled-coil containing protein kinase 1 (ROCK1), profilin1 (PFN1), and neuronal Wiskott-Aldrich Syndrome protein (N-WASP) expression, which was further reduced by haloperidol and clozapine. MK801 also increased the phosphorylated myosin light chain 2 (p-MLC2), postsynaptic density protein 95 (PSD-95), and c-jun expression, which was decreased by haloperidol and clozapine. p21 (RAC1-) activated kinase 1 (PAK1) expression was not affected by MK801.
\end{abstract}

\section{Introduction}

Antipsychotic drugs (APDs) have been used to ease psychotic symptoms for several decades. The main receptors known to bind APDs are dopamine and serotonin (5-HT) receptors [1], which have been shown to be related to psychotic diseases. Haloperidol, a typical antipsychotic drug, mainly binds the dopamine $\mathrm{D}_{2}$ receptor as well as the 5$\mathrm{HT}_{2 \mathrm{~A}}$ receptor [2]. Atypical APDs, such as clozapine and risperidone, mainly bind the $5-\mathrm{HT}_{2 \mathrm{~A}}$ receptor in addition to various other receptors [2]. Although the binding profiles of APDs and the biological effects of APD-bound receptors have been well studied, the detailed molecular mechanisms of APDs still need to be investigated. Haloperidol and clozapine can affect the phosphorylation of glycogen synthase kinase 3 (GSK3), extracellular signal-regulated kinase (ERK), and protein kinase B (Akt) and further modulate the ERK/AKT signaling pathway [3-7]. Various studies have also shown that haloperidol and clozapine might regulate protein kinase A (PKA) and cAMP-response elementbinding protein (CREB) $[8,9]$. Other studies have shown the differential effects of APDs in regulating apoptotic processes and oxidative stress signaling [10-13]. Neuronal plasticity in the brain has also been reported to be closely related to psychotic disorders, including schizophrenia. In various recent studies, APDs were also shown to regulate neuronal 
plasticity [14-16], which has been found to be regulated by the Rho signaling pathway [17-20]. These findings suggest that APDs might modulate neuronal cell plasticity by regulating Rho signaling pathway.

MK801 (dizocilpine) has been used to induce psychotic symptoms in various animal models to study the pathogenesis of schizophrenia. MK801 is an N-methyl-D-aspartate receptor (NMDA receptor, NMDAR) antagonist that can increase both positive and negative symptoms of schizophrenia in various abnormal behavior models [21, 22]. Social withdrawal, hyperactivity, and other psychotic behaviors have been observed in a number of studies using MK801 rodent models [23]. MK801-induced Ras homolog family member A (RhoA) and cell division control protein 42 (Cdc42) mRNA modulation in the rat brain have been investigated [24]. MK801 has been shown to regulate the Rho family protein expression and inhibit hippocampal neuron dendritic spine formation, ultimately impairing recognition in rats [24]. MK801 also diminishes neurite outgrowth, initiation, elongation, and branching in various types of neuronal cell cultures $[25,26]$. A recent study also showed that the alternation of the TAO kinase 2 (TAOK2) expression might induce neurodevelopmental and cognitive abnormalities by regulating the RhoA signaling [27]. The cytoplasmic Arabidopsis homolog of yeast Atg8 (AUT2) acts as an upstream regulator of Ras-related C3 botulinum toxin substrate 1 (Rac1), and Cdc42 in neurons regulates cytoskeletal dynamics, which might be related to cognitive memory and emotional control [28]. Aripiprazole, but not cannabidiol, olanzapine, or risperidone, has been shown to reverse MK801-induced deficiency in social recognition in rats [29]. In addition, olanzapine was shown to promote neurogenesis following MK801-induced cognitive impairment in mice $[30,31]$.

Rho signaling pathway modulates several biological functions in cells, including apoptotic gene expression, cell migration, cell morphological changes, and cytoskeleton reorganization. The RhoA protein activity is activated by RhoGDI1 and regulates Rho-associated coiled-coil containing protein kinase 1 (ROCK1) function to ultimately promote myosin activation and induce stress fiber contraction. Moreover, RhoA protein modulates actin-related protein 2/3 (ARP2/3) expression to regulate cell filopodium formation and cell migration. ARP2/3-mediated cytoskeletal remodeling is also modulated by Cdc42-induced p21 (RAC1-) activated kinase 1 (PAK1)/neuronal Wiskott-Aldrich Syndrome protein (N-WASP) activation [32]. PAK1 has been shown to be regulated by Rac1 signaling and to affect cell migration with Cdc42 [33-35]. Our previous findings suggested that APDs can induce RhoGDI pathway regulation to modulate cytoskeleton reorganization, which might be related to cell migration and dendritic spine formation in B35 neuronal cells [36]. Increased cell migration and F-actin condensation in B35 neuronal cells by haloperidol or clozapine treatment were also observed. To understand the effects of MK801 on RhoGDI signaling regulation in neuronal cells, we examined cell migration, F-actin condensation, and RhoGDI signaling regulation in MK801-treated B35 cells. We also treated MK801-treated B35 cells with haloperidol or clozapine to further clarify the roles of MK801, haloperidol, and clozapine in Rho signaling pathway regulation and downstream cytoskeleton regulation.

\section{Materials and Methods}

2.1. Antibodies. The antibodies used in this study were as follows: anti-ARP2/3, ab77084 (Abcam, Cambridge, UK); antiCdc42, ab41429 (Abcam); anti-c-jun, ab32137 (Abcam); antiphosphorylated myosin light chain 2 (p-MLC2, phospho-MLC2) (Ser19), \#3671 (Cell Signaling Danvers, MA, US); anti-N-WASP, \#4848 (Cell Signaling); anti-PAK1, ab40852 (Abcam); anti-Profilin-1, \#3237 (Cell Signaling); antipostsynaptic density protein 95 (PSD-95), \#3450 (Cell Signaling); anti-RhoA, ab54835 (Abcam); anti-RhoGDI1, ab118159 (Abcam); anti-ROCK1, ab45171 (Abcam); and antiactin, ab6276 (Abcam). All antibodies were used at the fold dilution recommended by the manufacturer.

2.2. Cell Culture and APD Treatments. MK801, haloperidol, and clozapine were obtained from Sigma-Aldrich (St. Louis, USA). B35 cells were purchased from the Bioresource Collection and Research Center (BCRC) of the Food Industry Research and Development Institute (FRDI), Taiwan. B35 cells were maintained in MEM (Invitrogen, Life Technologies) supplemented with 10\% fetal bovine serum (Invitrogen, Life Technologies), $2 \mathrm{mM}$ L-glutamine, $100 \mathrm{U} / \mathrm{ml}$ penicillin, and $100 \mu \mathrm{g} / \mathrm{ml}$ streptomycin. MK801 was added to the medium daily at a final concentration of $25 \mu \mathrm{M}$ for 7 days. On the 8th day, MK801 and either haloperidol or clozapine, at final concentrations of $4 \mu \mathrm{g} / \mathrm{ml}(10 \mu \mathrm{M})$ for haloperidol and $2.5 \mu \mathrm{g} / \mathrm{ml}(7.65 \mu \mathrm{M})$ for clozapine, were added to the medium daily for 7 days to examine whether haloperidol or clozapine reversed the effects of MK801. After 7 days of MK801/APD treatment, B35 cells were harvested to examine the expression levels of RhoGDI, RhoA, CDC42, ROCK1, pMLC2, PFN1, N-WASP, ARP2/3, PAK1, c-jun, and PSD-95.

2.3. Western Blot Analysis. The total cell extracts of B35 cells were prepared by lysing cells in mammalian protein extraction buffer (GE Healthcare Bio-Sciences, Uppsala, Sweden) containing Protease Inhibitor Mix (GE Healthcare Bio-Sciences) and phosphatase inhibitors $(2 \mathrm{mM} \mathrm{NaF}$ and $1 \mathrm{mM}$ $\mathrm{Na}_{3} \mathrm{VO}_{4}$ ). A total of $10-50 \mu \mathrm{g}$ total protein from B35 cell extracts was separated on $10-15 \%$ polyacrylamide gels containing sodium dodecyl sulfate. Proteins in the gel were transferred to polyvinylidene difluoride membranes, and the membranes were blocked by Membrane Blocking Solution (Life Technology, Frederick, MD, USA) for $1 \mathrm{~h}$. The membranes were then incubated with specific primary antibodies for $12 \mathrm{~h}$ at $4^{\circ} \mathrm{C}$ followed by horseradish peroxidaseconjugated goat antimouse or antirabbit antibodies (Cat. nos. 401215 and 401315, Calbiochem, Darmstadt, Germany) at room temperature for $4 \mathrm{~h}$. The protein bands were developed by using the Amersham ECL kit (Amersham, Bucks, UK). Beta-actin was used as a quantification control.

2.4. Cell Migration Assay. B35 cells were treated with MK801 for 7 days followed by 5 days of treatment with MK801 combined with haloperidol or clozapine. Drug-treated B35 cells $\left(5 \times 10^{3} /\right.$ well $)$ were seeded into the upper compartment of 
an $8-\mu \mathrm{m}$ pore polycarbonate membrane Transwell insert (Costar, Corning Incorporation, Kennebunk, ME, USA) in a 24-well tissue culture plate. Culture medium $(0.7 \mathrm{ml})$ containing MK801 and either haloperidol or clozapine were added to the lower well. After a 48-h incubation, B35 cells were fixed with methanol and then stained with a $50 \mu \mathrm{g} / \mathrm{ml}$ solution of propidium iodide (Sigma) for $30 \mathrm{~min}$ at room temperature. The number of cells on each membrane was counted on a microscope at 40x magnification. All experiments were performed in triplicate.

2.5. Actin Filament (F-actin) Staining of B35 Cells. B35 cells were treated with MK801 for 7 days followed by a 5-day treatment of MK801 in combination with haloperidol or clozapine. A total of $5 \times 10^{3}$ drug-treated B35 cells were seeded on coverslips in a 6-well plate and cultured in medium with MK801 or haloperidol/clozapine for another 2 days. After drug treatment, the coverslips with B35 cells were transferred to a fresh 6-well plate and washed with $1 \mathrm{X}$ PBS. The cells were then fixed by incubating the coverslips in methanol, washed with $1 \mathrm{X}$ PBS, and stained with $1 \mathrm{X}$ phalloidin solution (CytoPainter Phalloidin-iFluor 488 Reagent, ab176753 $(\mathrm{Abcam}))$ at room temperature for $90 \mathrm{~min}$. The excess phalloidin was removed by washing the cells with PBS 2-3 times. The coverslips were then mounted and sealed onto glass slides with SlowFade ${ }^{\mathrm{TM}}$ Diamond Antifade Mountant with DAPI (Invitrogen, Life Technologies Incorporation, Eugene, OR, USA) and imaged on a microscope.

2.6. Quantification and Statistical Analysis. Cell migration assays and differences in normalized protein expression levels between B35 cells treated with different drugs were analyzed by using one-way ANOVA followed by Dunnett's post hoc comparison test. One-way ANOVA and Dunnett's post hoc comparison test were performed with SPSS Statistics 17.0. Significant differences were defined as those with a $p$ value less than $0.05\left(^{*}\right)$ or $0.01\left({ }^{* *}\right)$.

\section{Results}

3.1. Haloperidol and Clozapine Reverse MK801-Induced Rho Family Protein Regulation. In line with our previous findings, both haloperidol and clozapine increased the CDC42 expression levels but did not affect the RhoA expression in B35 cells. Haloperidol, but not clozapine, induced Racl expression in B35 cells. Figure 1 shows that MK801 increased the RhoGDI1 ( $p$ value $<0.05)$ and RhoA $(p$ value $<0.01)$ expression and reduced the CDC42 ( $p$ value $<0.01$ ) and Racl ( $p$ value $<0.01)$ expression in B35 cells. The increased RhoGDI1 expression induced by MK801 in B35 cells was reduced by haloperidol ( $p$ value $<0.05$ ) or clozapine $(p$ value $<0.05)$ treatment. The MK801-induced increase in the RhoA expression was reduced by haloperidol $(p$ value $<0.01)$ or clozapine $(p$ value $<0.01)$. At the same time, the reduction in CDC42 expression induced by MK801 was reversed in B35 cells treated with haloperidol ( $p$ value $<0.01$ ) or clozapine ( $p$ value $<0.01)$. The MK801-induced reduction in the Rac1 expression was reversed by haloperidol $(p$ value $<0.01)$ or clozapine ( $p$ value $<0.05)$.
3.2. MK801 Modulated RhoA-Related Signaling and F-actin Condensation. F-actin condensation is thought to be regulated by RhoA signaling, including ROCK1, p-MLC2, and PFN1 modulation. F-actin condensation in B35 cells was inhibited by the MK801 treatment and restored by treating the cells with haloperidol or clozapine (Figure 2). At the same time, we investigated whether the expression levels of ROCK1 ( $p$ value $<0.05)$ and PFN1 ( $p$ value $<0.05)$ were reduced by MK801 in B35 cells (Figure 3). MK801 reduced the ROCK1 expression, which was further decreased by haloperidol ( $p$ value $<0.05)$ or clozapine $(p$ value $<0.05)$. The PFN1 expression was reduced by MK801 and further downregulated by haloperidol $(p$ value $<0.01)$ and clozapine $(p$ value $<0.05)$. In contrast, the increase in $\mathrm{p}-\mathrm{MLC} 2$ induced by MK801 ( $p$ value $<0.01$ ) in B35 cells could be reversed by haloperidol $(p$ value $<0.05)$ or clozapine $(p$ value $<0.05)$ treatment.

3.3. Haloperidol/Clozapine Modulated MK801-Induced Cell Migration Inhibition in B35 Cells. To reveal the relationship between MK801- and haloperidol-/clozapine-modulated cell migration ability in B35 cells, $5 \times 10^{3}$ drug-treated B35 cells were seeded into the insert of a Transwell plate and treated with MK801, haloperidol, or clozapine for another $24 \mathrm{~h}$. Migrated B35 cells were stained and counted to analyze the regulation of the migration ability of B35 cells. Figure 4 shows that the migration ability of B35 cells was inhibited by 14 days of MK801 treatment ( $p$ value $<0.01$ ). The inhibitory effects of MK801 on cell migration could be significantly reversed by treating cells with haloperidol ( $p$ value $<0.01$ ) or clozapine for 7 days ( $p$ value $<0.01$ ).

3.4. Haloperidol/Clozapine Induced Changes in CDC42 and Rac1 Signaling in MK801-Treated B35 Cells. CDC42 and Rac1 signaling, which modulate filopodium formation and cell migration, were investigated. As shown in Figure 5, the $\mathrm{N}-$ WASP expression in B35 cells was downregulated by MK801 treatment ( $p$ value $<0.05)$. N-WASP expression in MK801-treated B35 cells was further inhibited by haloperidol ( $p$ value $<0.05)$ or clozapine $(p$ value $<0.05)$ treatment. We also investigated whether the PAK1 expression in B35 cells is affected by the MK801 treatment. The PAK1 expression in MK801-treated B35 cells was reduced by haloperidol or clozapine. Furthermore, the ARP2/3 expression was reduced by the MK801 $(p$ value $<0.01)$ treatment in B35 cells. The MK801-induced downregulation of the ARP2/3 expression in B35 cells was rescued by haloperidol ( $p$ value $<0.05$ ) or clozapine ( $p$ value $<0.05)$.

3.5. Haloperidol/Clozapine Modulated c-jun and PSD-95 Expression in MK801-Treated B35 Cells. Rho family proteins have been found to be related to dendritic spine morphology, cytoskeleton reorganization, and apoptotic gene expression regulation. As shown in Figure 6, the increased expression of PSD-95 was induced by MK801 ( $p$ value <0.01) in B35 cells. The MK801-induced increase in the PSD-95 expression could be reversed by haloperidol ( $p$ value $<0.05$ ) or clozapine ( $p$ value $<0.01)$. The $c$-jun expression in B35 cells was increased by MK801 ( $p$ value $<0.01$ ), whereas haloperidol 


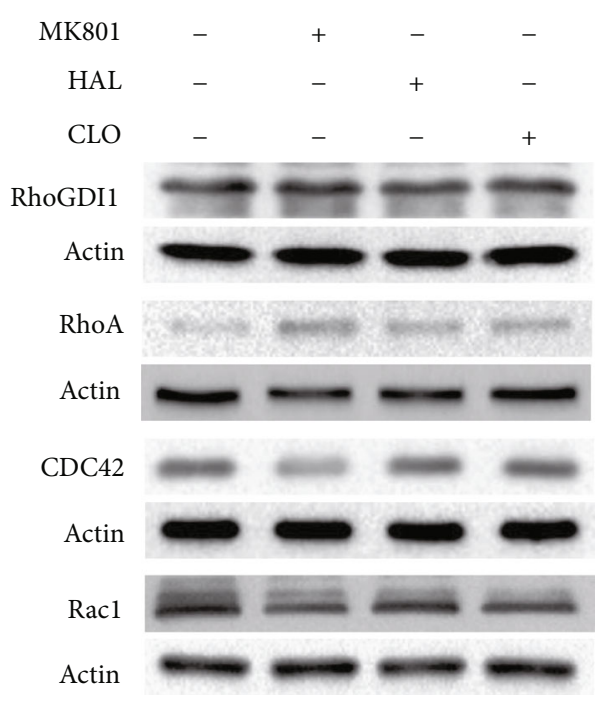

(a)

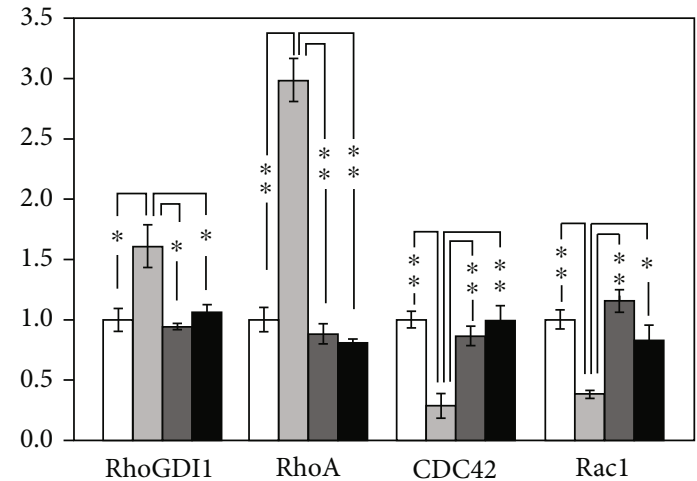

$\square$ Control

$\square$ MK801

$\square$ MK801+HAL

- MK801+CLO

(b)

Figure 1: Haloperidol (HAL) and clozapine (CLO) reverse the MK801-induced Rho family protein regulation. (a) Western blot revealing APD-induced changes in the expression of the RhoGDI, RhoA, CDC42, and Rac1 proteins in B35 cells. (b) Protein expression was quantified and normalized to beta-actin, and the relative expression of each protein shown in the bar chart was calculated from triplicate western blot data that were obtained from three different batches of APD-treated cells and analyzed using ANOVA followed by Dunnett's test $\left({ }^{*} p\right.$ value $<0.05 ;{ }^{* *} p$ value $\left.<0.01\right)$.

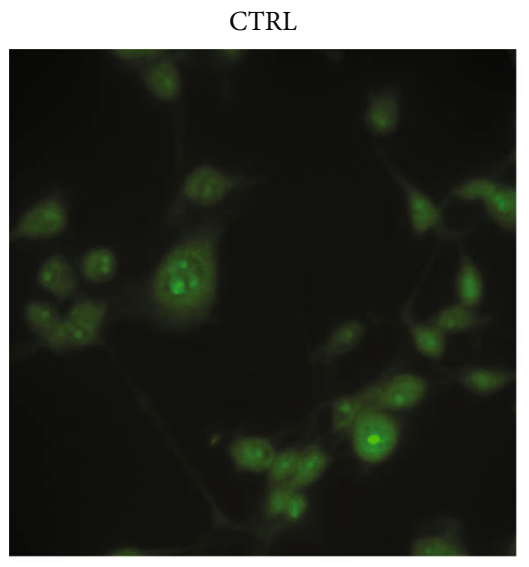

MK-801+HAL

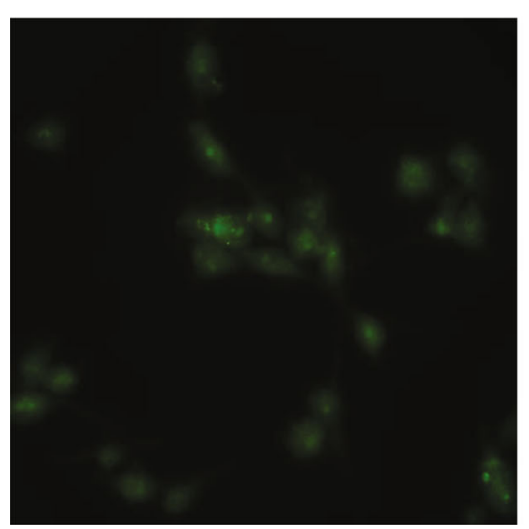

MK-801

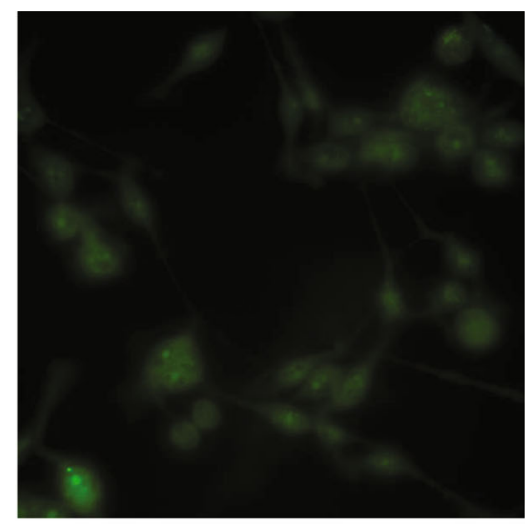

MK-801+CLO

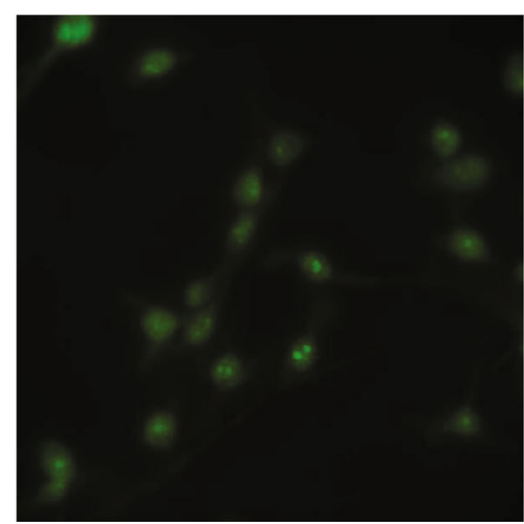

Figure 2: Effects of MK801, haloperidol, and clozapine on F-actin condensation in B35 cells. The B35 cells were treated with MK801 followed by haloperidol (HAL) or clozapine (CLO) treatment. CytoPainter Phalloidin-iFluor 488 Reagent was used to stain the F-actin of B35 cells. 


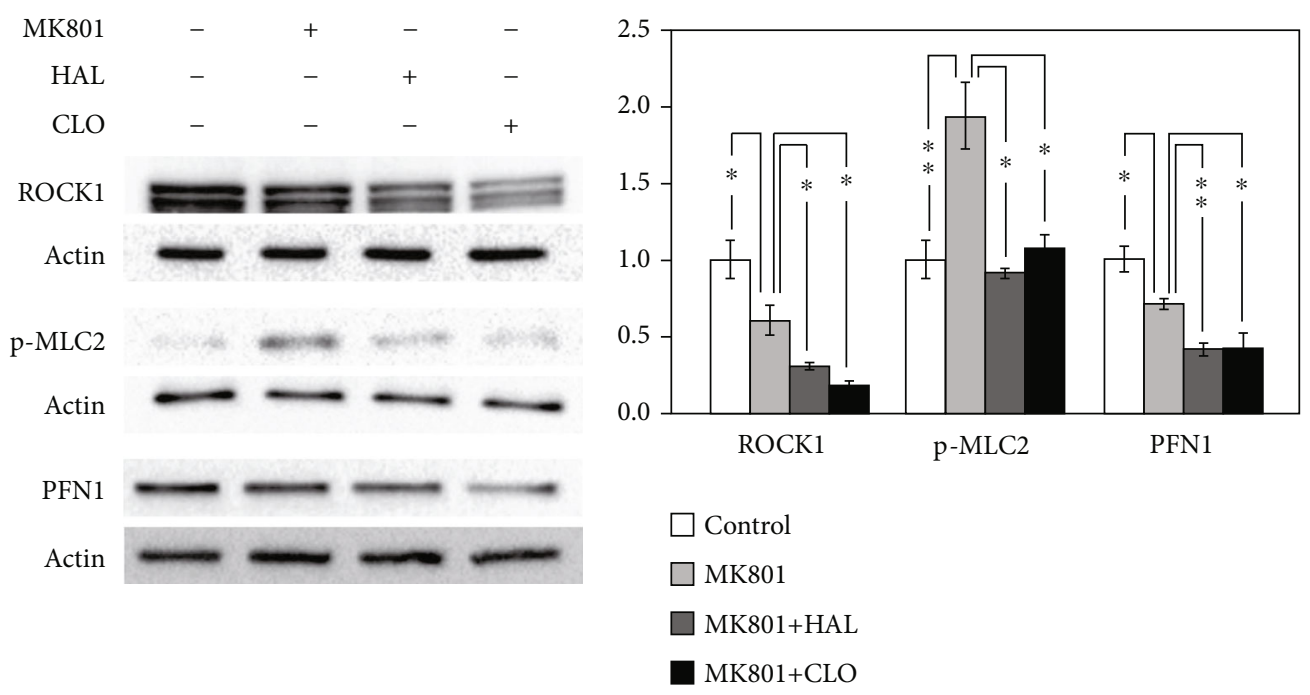

(a)

(b)

Figure 3: Effects of MK801, haloperidol (HAL), and clozapine (CLO) on ROCK1, p-MLC2, and PFN1 regulation in B35 cells. (a) Western blot revealing APD-induced changes in the expression of ROCK1, p-MLC2, and PFN1 proteins in B35 cells. (b) Protein expression was quantified and normalized to beta-actin, and the relative expression of each protein in the bar chart was calculated from triplicate western blot data obtained from three different batches of APD-treated cells and analyzed using ANOVA followed by Dunnett's test $\left({ }^{*} p\right.$ value $<0.05 ;{ }^{* *} p$ value $\left.<0.01\right)$.
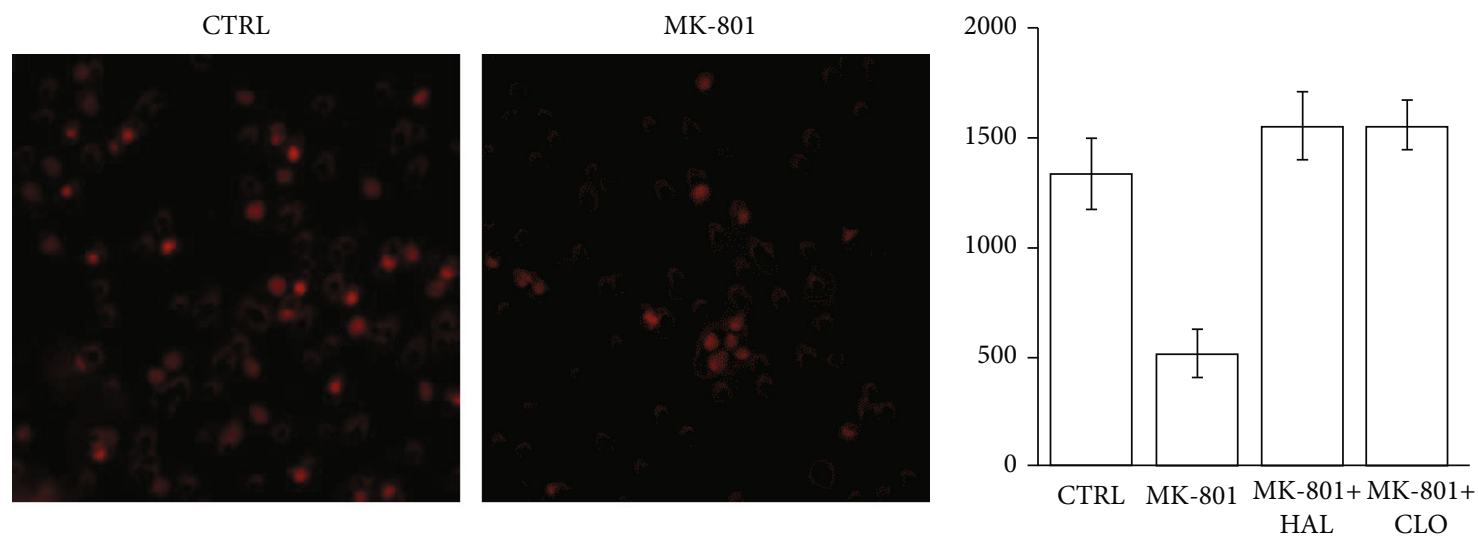

MK-801+HAL

MK-801+CLO
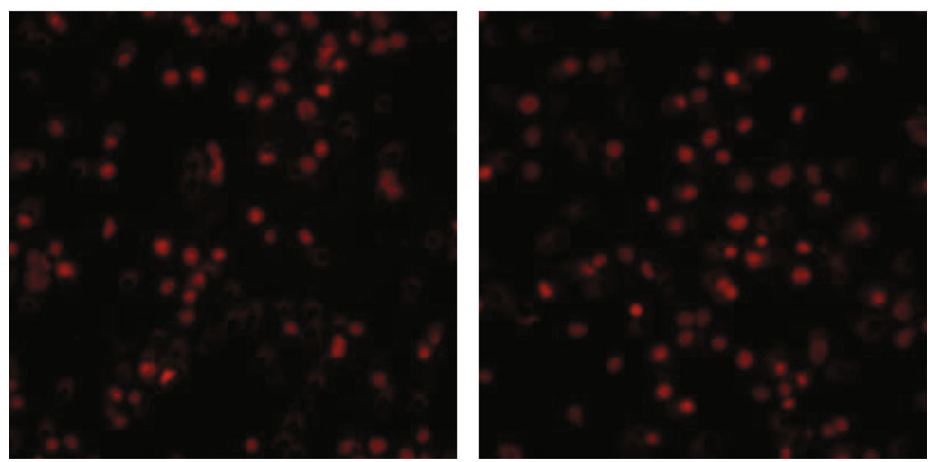

(a)

(b)

Figure 4: Effects of MK801, haloperidol (HAL), and clozapine (CLO) on the cell migration ability of B35 cells. (a) Cell migration was analyzed, and the cell numbers were counted after staining. (b) The bar chart was constructed from triplicate cell count data obtained from three different batches of MK801/APD-treated cells and analyzed using ANOVA followed by Dunnett's test $\left({ }^{*} p\right.$ value $<0.05$; ${ }^{* *} p$ value $<0.01)$. 


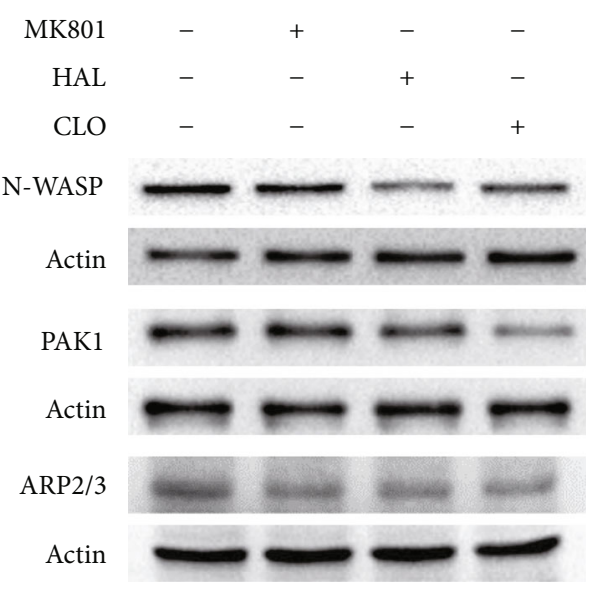

(a)

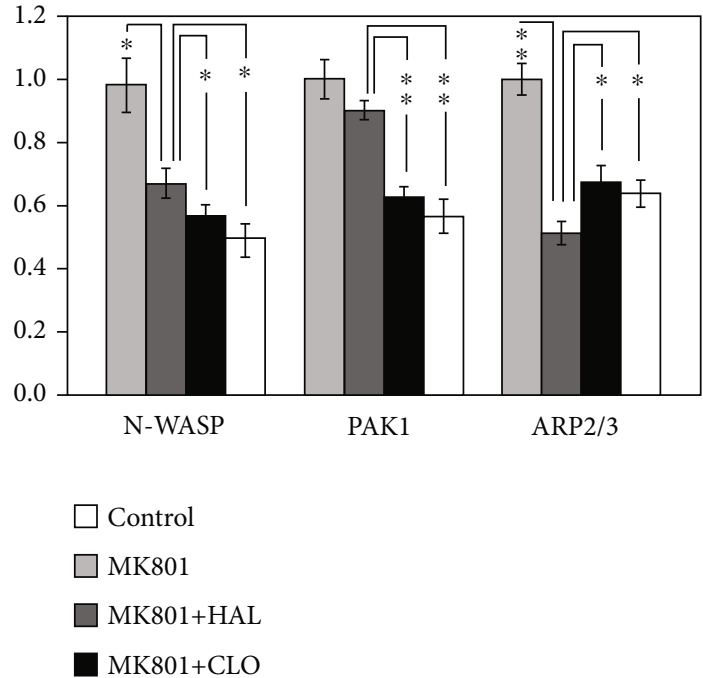

(b)

FIGURE 5: Effects of MK801, haloperidol (HAL), and clozapine (CLO) on N-WASP, PAK1, and ARP2/3 regulation in B35 cells. (a) Western blot revealing APD-induced changes in the expression of the N-WASP, PAK1, and ARP2/3 proteins in B35 cells. (b) Protein expression was quantified and normalized to beta-actin, and the relative expression of each protein chart bar was constructed from triplicate western blot data obtained from three different batches of APD-treated cells using ANOVA followed by Dunnett's test $\left({ }^{*} p\right.$ value $<0.05$; ${ }^{* *} p$ value $\left.<0.01\right)$.

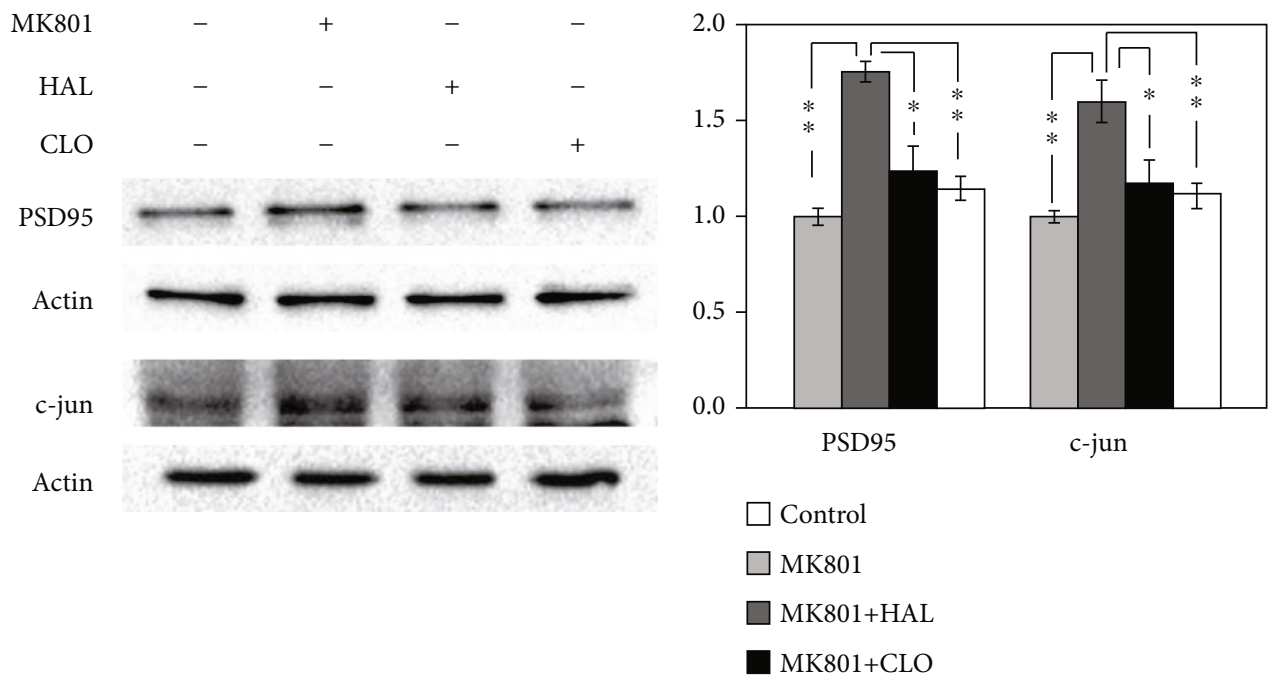

(a)

(b)

FIGURE 6: Effects of MK801, haloperidol (HAL), and clozapine (CLO) on PSD95 and c-jun regulation in B35 cells. (a) Western blot revealing APD-induced changes in the expression of PSD-95 and c-jun proteins in B35 cells. (b) Protein expression was quantified and normalized to beta-actin, and the relative expression of each protein in the bar chart was calculated from triplicate western blot data obtained from three different batches of APD-treated cells and analyzed using ANOVA followed by Dunnett's test $\left({ }^{*} p\right.$ value $<0.05 ;{ }^{* *} p$ value $\left.<0.01\right)$.

( $p$ value $<0.05)$ and clozapine $(p$ value $<0.01)$ reversed the effect of MK801 on c-jun expression in B35 cells.

\section{Discussion}

The present study revealed that MK801 could modulate RhoGDI1, Rho family proteins, and Rho family proteinrelated signaling in B35 cells. MK801 could also regulate the F-actin remodeling and the cell migration ability of B35 cells. The antipsychotic drugs haloperidol and clozapine dif- ferentially modulated the MK801-induced effects and the Rho signaling pathway in B35 cells. MK801 has been reported to induce impairments in learning and memory in mice and rats. MK801 is an antagonist of NMDA receptors and affects learning, cognition, and memory in animals by modulating the synaptic plasticity of neuronal cells in the brain [37-39]. Various studies have also shown that MK801 might modulate synaptic plasticity and postsynaptic dendrite morphogenesis by regulating the activity of Rho family proteins, including RhoA, Cdc42, and Racl [40-42]. The results 
of this study also showed that MK801-induced increases in the expression of RhoGDI1 and RhoA can be reversed by either haloperidol or clozapine. Moreover, haloperidol and clozapine could reverse the MK801-induced inhibition of CDC42 and Rac1 in B35 cells. These results suggested that the drug-induced regulation of the Rho signaling pathway might be the key to modulating MK801-induced abnormal behaviors.

ROCK1 and PAK1 are two downstream proteins in the Rho signaling pathway that have been shown to be important regulators of synaptic plasticity in maintaining the dendritic spine actin cytoskeleton $[43,44]$. In this study, we found that both haloperidol and clozapine could reverse MK801induced F-actin separation. Additionally, the MK801induced activation of the MLC2 (p-MLC2) expression in B35 cells could be diminished by haloperidol or clozapine treatment. This result suggested that activated MLC2 might be an important target for haloperidol or clozapine in regulating the F-actin condensation in MK801-treated B35 cells. The increased p-MLC2 expression might be caused by compensatory induction due to the decrease in F-actin condensation in MK801-treated B35 cells.

We also showed that both haloperidol and clozapine could recover MK801-induced cell migration reduction in B35 cells. Moreover, the decreased ARP2/3 expression induced by MK801 could be reversed by the haloperidol or clozapine treatment in B35 cells. N-WASP and ARP2/3 are reported to be critical regulators of the development of dendritic spines and synapses [45]. Various studies have also described the roles of N-WASP, PAK1, and ARP2/3 in the cell migration of neuronal cells [46-48]. The results of this study indicated that ARP2/3 might be a key target of haloperidol and clozapine through which they modulate MK801induced cell migration inhibition in B35 cells. Previous reports have suggested that Wiskott-Aldrich syndrome protein (WASP-) interacting SH3 protein (WISH), a novel neural protein, can activate the Arp $2 / 3$ complex through both $\mathrm{N}$ WASP-dependent and N-WASP-independent pathways without CDC42 [49, 50]. Activation of AMPARs and NMDARs has been proposed to trigger cytoskeletal reorganization through Rho GTPases (RhoA, CDC42, and Rac1) in cells [51]. AMPARs and NMDARs are also closely related to the PSD-95 expression and dendrite spine formation. It has been suggested that ARP2/3 can induce enlargement of the spine head [52]. These research results suggested that the regulation of PSD-95 might be related to the CDC42mediated modulation of N-WASP, PAK1, and ARP2/3 function. Our data also showed that the ROCK1, N-WASP, PAK1, and PFN1 expression levels are not consistent with the Rho family protein expression, drug-induced cell migration, or F-actin condensation. We propose that the expression or activation of ROCK1, N-WASP, PAK1, and PFN1 could be controlled by factors other than the Rho family proteins in this study. The detailed mechanisms by which the ROCK1, N-WASP, PAK1, and PFN1 expression are regulated should be further investigated.

Except for the regulation of cell migration, F-actin condensation, Rho signaling pathway, and PSD-95 expression, the c-jun expression induced by MK801 could be reversely regulated by haloperidol or clozapine in B35 cells. This result suggested that the MK801-induced modulation of apoptotic gene expression could be reversed by haloperidol or clozapine treatment in B35 cells. The modulation of gene expression in neuronal cells might also regulate cell functions to fine-tune neuronal cell activity.

\section{Conclusion}

Previous studies have shown that MK801-induced abnormal behavior is linked to the Rho family protein regulation. Our study discovered that the MK801-induced abnormalities in cell migration, F-actin reorganization, and PSD-95 expression could be recovered by haloperidol- or clozapineinduced regulation of the Rho signaling pathway. These results suggested that the Rho signaling pathway might be one of the regulatory factors that can modulate MK801induced abnormal behaviors.

\section{Data Availability}

All the data used to support the findings of this study are available from the corresponding author upon request.

\section{Disclosure}

All sponsors or funders play no role in the study design, data collection and analysis, decision to publish, or the preparation of the manuscript.

\section{Conflicts of Interest}

The authors declare that there is no conflict of interest regarding the publication of this paper.

\section{Acknowledgments}

The authors thank the Core Laboratory of the Taipei Tzu Chi Hospital, Buddhist Tzu Chi Medical Foundation for support. This work was supported by the grant from the Taipei Tzu Chi Hospital, Buddhist Tzu Chi Medical Foundation, New Taipei City, Taiwan (TCRD-TPE-108-13).

\section{References}

[1] I. Kusumi, S. Boku, and Y. Takahashi, "Psychopharmacology of atypical antipsychotic drugs: from the receptor binding profile to neuroprotection and neurogenesis," Psychiatry and Clinical Neurosciences, vol. 69, no. 5, pp. 243-258, 2015.

[2] F. P. Bymaster, D. O. Calligaro, J. F. Falcone et al., "Radioreceptor binding profile of the atypical antipsychotic olanzapine," Neuropsychopharmacology, vol. 14, no. 2, pp. 87-96, 1996.

[3] X. H. Lu and D. S. Dwyer, "Second-generation antipsychotic drugs, olanzapine, quetiapine, and clozapine enhance neurite outgrowth in PC12 cells via PI3K/AKT, ERK, and pertussis toxin-sensitive pathways," Journal of Molecular Neuroscience, vol. 27, no. 1, pp. 043-064, 2005.

[4] B. H. Yang, H. Son, S. H. Kim, J. H. Nam, J. H. Choi, and J. S. Lee, "Phosphorylation of ERK and CREB in cultured hippocampal neurons after haloperidol and risperidone 
administration," Psychiatry and Clinical Neurosciences, vol. 58, no. 3, pp. 262-267, 2004.

[5] W. Ukai, H. Ozawa, M. Tateno, E. Hashimoto, and T. Saito, "Neurotoxic potential of haloperidol in comparison with risperidone: implication of Akt-mediated signal changes by haloperidol," Journal of Neural Transmission (Vienna), vol. 111, no. 6, pp. 667-681, 2004.

[6] H. Alimohamad, N. Rajakumar, Y. H. Seah, and W. Rushlow, "Antipsychotics alter the protein expression levels of beta-catenin and GSK-3 in the rat medial prefrontal cortex and striatum," Biological Psychiatry, vol. 57, no. 5, pp. 533-542, 2005.

[7] X. Li, K. M. Rosborough, A. B. Friedman, W. Zhu, and K. A. Roth, "Regulation of mouse brain glycogen synthase kinase-3 by atypical antipsychotics," The International Journal of Neuropsychopharmacology, vol. 10, no. 1, pp. 7-19, 2007.

[8] L. Pozzi, K. Håkansson, A. Usiello et al., "Opposite regulation by typical and atypical anti-psychotics of ERK1/2, CREB and Elk-1 phosphorylation in mouse dorsal striatum," Journal of Neurochemistry, vol. 86, no. 2, pp. 451-459, 2003.

[9] A. V. Turalba, K. A. Leite-Morris, and G. B. Kaplan, "Antipsychotics regulate cyclic AMP-dependent protein kinase and phosphorylated cyclic AMP response element-binding protein in striatal and cortical brain regions in mice," Neuroscience Letters, vol. 357, no. 1, pp. 53-57, 2004.

[10] S. I. Deutsch, R. B. Rosse, B. L. Schwartz, and J. Mastropaolo, "A revised excitotoxic hypothesis of schizophrenia: therapeutic implications," Clinical Neuropharmacology, vol. 24, no. 1, pp. 43-49, 2001.

[11] M. Lundberg, S. Curbo, H. Bohman et al., "Clozapine protects adult neural stem cells from ketamine-induced cell death in correlation with decreased apoptosis and autophagy," Bioscience Reports, vol. 40, no. 1, 2020.

[12] H. M. An, Y. L. Tan, J. Shi et al., "Ginkgo biloba leaf extract and alpha-tocopherol attenuate haloperidol-induced orofacial dyskinesia in rats: possible implication of antiapoptotic mechanisms by preventing Bcl-2 decrease and Bax elevation," Phytomedicine, vol. 23, no. 13, pp. 1653-1660, 2016.

[13] A. T. Chen and H. A. Nasrallah, "Neuroprotective effects of the second generation antipsychotics," Schizophrenia Research, vol. 208, pp. 1-7, 2019.

[14] M. Morais, P. Patrício, A. Mateus-Pinheiro et al., "The modulation of adult neuroplasticity is involved in the moodimproving actions of atypical antipsychotics in an animal model of depression," Translational Psychiatry, vol. 7, no. 6, article e1146, 2017.

[15] H. Ozdemir, A. Ertugrul, K. Basar, and E. Saka, "Differential effects of antipsychotics on hippocampal presynaptic protein expressions and recognition memory in a schizophrenia model in mice," Progress in Neuro-Psychopharmacology \& Biological Psychiatry, vol. 39, no. 1, pp. 62-68, 2012.

[16] A. S. C. França, B. Lobão-Soares, L. Muratori et al., "D2 dopamine receptor regulation of learning, sleep and plasticity," European Neuropsychopharmacology, vol. 25, no. 4, pp. 493504, 2015.

[17] K. M. Greathouse, B. W. Henderson, E. G. Gentry, and J. H. Herskowitz, "Fasudil or genetic depletion of ROCK1 or ROCK2 induces anxiety-like behaviors," Behavioural Brain Research, vol. 373, article 112083, 2019.

[18] S. Jiang, Z. Hao, X. Li et al., "Ketamine destabilizes growth of dendritic spines in developing hippocampal neurons in vitro via a Rho-dependent mechanism," Molecular Medicine Reports, vol. 18, no. 6, pp. 5037-5043, 2018.

[19] M. E. Fox, R. Chandra, M. S. Menken et al., "Dendritic remodeling of D1 neurons by RhoA/Rho-kinase mediates depression-like behavior," Molecular Psychiatry, vol. 25, no. 5, pp. 1022-1034, 2020.

[20] K. M. Greathouse, B. D. Boros, J. F. Deslauriers et al., "Distinct and complementary functions of rho kinase isoforms ROCK1 and ROCK2 in prefrontal cortex structural plasticity," Brain Structure \& Function, vol. 223, no. 9, pp. 4227-4241, 2018.

[21] J. P. Rung, A. Carlsson, K. Ryden Markinhuhta, and M. L. Carlsson, "(+)-MK-801 induced social withdrawal in rats; a model for negative symptoms of schizophrenia," Progress in Neuro-Psychopharmacology \& Biological Psychiatry, vol. 29, no. 5, pp. 827-832, 2005.

[22] D. Manahan-Vaughan, D. von Haebler, C. Winter, G. Juckel, and U. Heinemann, "A single application of MK801 causes symptoms of acute psychosis, deficits in spatial memory, and impairment of synaptic plasticity in rats," Hippocampus, vol. 18, no. 2, pp. 125-134, 2008.

[23] V. Bubenikova-Valesova, J. Horacek, M. Vrajova, and C. Hoschl, "Models of schizophrenia in humans and animals based on inhibition of NMDA receptors," Neuroscience and Biobehavioral Reviews, vol. 32, no. 5, pp. 1014-1023, 2008.

[24] D. Han, L. Xu, H. Xiao, G. C. Prado Schmidt, and S. Shi, “Dizocilpine reduces head diameter of dendritic spines in the hippocampus of adolescent rats," Psychiatry Research, vol. 210, no. 1, pp. 351-356, 2013.

[25] R. Cuppini, S. Sartini, P. Ambrogini, E. Falcieri, M. C. Maltarello, and G. Gallo, "Control of neuron outgrowth by NMDA receptors," Journal of Submicroscopic Cytology and Pathology, vol. 31, no. 1, pp. 31-40, 1999.

[26] L. Klimaviciusa, D. Safiulina, A. Kaasik, V. Klusa, and A. Zharkovsky, "The effects of glutamate receptor antagonists on cerebellar granule cell survival and development," Neurotoxicology, vol. 29, no. 1, pp. 101-108, 2008.

[27] M. Richter, N. Murtaza, R. Scharrenberg et al., “Altered TAOK2 activity causes autism-related neurodevelopmental and cognitive abnormalities through RhoA signaling," Molecular Psychiatry, vol. 24, no. 9, pp. 1329-1350, 2019.

[28] K. Hori, T. Nagai, W. Shan et al., "Cytoskeletal regulation by AUTS2 in neuronal migration and neuritogenesis," Cell Reports, vol. 9, no. 6, pp. 2166-2179, 2014.

[29] S. Deiana, A. Watanabe, Y. Yamasaki et al., "MK-801-induced deficits in social recognition in rats: reversal by aripiprazole, but not olanzapine, risperidone, or cannabidiol," Behavioural Pharmacology, vol. 26, no. 8, pp. 748-765, 2015.

[30] J. C. Song, M. K. Seo, S. W. Park, J. G. Lee, and Y. H. Kim, “Differential effects of olanzapine and haloperidol on MK-801induced memory impairment in mice," Clinical Psychopharmacology and Neuroscience, vol. 14, no. 3, pp. 279-285, 2016.

[31] X. Liu, J. Li, C. Guo et al., "Olanzapine reverses MK-801induced cognitive deficits and region-specific alterations of NMDA receptor subunits," Frontiers in Behavioral Neuroscience, vol. 11, p. 260, 2018.

[32] M. Parsons, J. Monypenny, S. M. Ameer-Beg et al., "Spatially distinct binding of Cdc42 to PAK1 and N-WASP in breast carcinoma cells," Molecular and Cellular Biology, vol. 25, no. 5, pp. 1680-1695, 2005.

[33] H. Imamura, K. Takaishi, K. Nakano et al., "Rho and Rab small $\mathrm{G}$ proteins coordinately reorganize stress fibers and focal 
adhesions in MDCK cells," Molecular Biology of the Cell, vol. 9, no. 9, pp. 2561-2575, 1998.

[34] M. A. Sells, J. T. Boyd, and J. Chernoff, "p21-activated kinase 1 (Pak1) regulates cell motility in mammalian fibroblasts," The Journal of Cell Biology, vol. 145, no. 4, pp. 837-849, 1999.

[35] M. A. Del Pozo, W. B. Kiosses, N. B. Alderson, N. Meller, K. M. Hahn, and M. A. Schwartz, "Integrins regulate GTP-Rac localized effector interactions through dissociation of Rho-GDI," Nature Cell Biology, vol. 4, no. 3, pp. 232-239, 2002.

[36] M. L. Chen, F. M. Tsai, M. C. Lee, and Y. Y. Lin, "Antipsychotic drugs induce cell cytoskeleton reorganization in glial and neuronal cells via Rho/Cdc42 signal pathway," Progress in Neuro-Psychopharmacology \& Biological Psychiatry, vol. 71, pp. 14-26, 2016.

[37] G. J. Kant, W. L. Wright, T. N. Robinson III, and C. P. D'Angelo, "Effects of MK-801 on learning and memory as assessed using a novel water maze," Pharmacology, Biochemistry, and Behavior, vol. 39, no. 2, pp. 479-485, 1991.

[38] F. J. van der Staay, K. Rutten, C. Erb, and A. Blokland, "Effects of the cognition impairer MK-801 on learning and memory in mice and rats," Behavioural Brain Research, vol. 220, no. 1, pp. 215-229, 2011.

[39] Y. Humeau and D. Choquet, "The next generation of approaches to investigate the link between synaptic plasticity and learning," Nature Neuroscience, vol. 22, no. 10, pp. 1536-1543, 2019.

[40] V. Schubert and C. G. Dotti, "Transmitting on actin: synaptic control of dendritic architecture," Journal of Cell Science, vol. 120, no. 2, pp. 205-212, 2007.

[41] Y. Chen, Z. Liang, E. Fei et al., "Axin regulates dendritic spine morphogenesis through Cdc42-dependent signaling," PLoS One, vol. 10, no. 7, article e0133115, 2015.

[42] M. Perez-Rando, E. Castillo-Gómez, R. Guirado et al., "NMDA receptors regulate the structural plasticity of spines and axonal boutons in hippocampal interneurons," Frontiers in Cellular Neuroscience, vol. 11, p. 166, 2017.

[43] A. Salminen, T. Suuronen, and K. Kaarniranta, "ROCK, PAK, and Toll of synapses in Alzheimer's disease," Biochemical and Biophysical Research Communications, vol. 371, no. 4, pp. 587-590, 2008.

[44] J. Yan, Y. Pan, X. Zheng et al., "Comparative study of ROCK1 and ROCK2 in hippocampal spine formation and synaptic function," Neuroscience Bulletin, vol. 35, no. 4, pp. 649-660, 2019.

[45] A. M. Wegner, C. A. Nebhan, L. Hu et al., "N-wasp and the arp2/3 complex are critical regulators of actin in the development of dendritic spines and synapses," The Journal of Biological Chemistry, vol. 283, no. 23, pp. 15912-15920, 2008.

[46] X. Pan, X. Chang, C. Leung et al., "PAK1 regulates cortical development via promoting neuronal migration and progenitor cell proliferation," Molecular Brain, vol. 8, no. 1, p. 36, 2015.

[47] S. S. Park, M. O. Kim, S. P. Yun et al., " $\mathrm{C}_{16}$-Ceramide-induced $\mathrm{F}$-actin regulation stimulates mouse embryonic stem cell migration: involvement of N-WASP/Cdc42/Arp2/3 complex and cofilin-1/ $\alpha$-actinin," Biochimica et Biophysica Acta (BBA) - Molecular and Cell Biology of Lipids, vol. 1831, no. 2, pp. 350-360, 2013.

[48] B. J. Perrin, K. J. Amann, and A. Huttenlocher, "Proteolysis of cortactin by calpain regulates membrane protrusion during cell migration," Molecular Biology of the Cell, vol. 17, no. 1, pp. 239-250, 2006.

[49] M. Fukuoka, S. Suetsugu, H. Miki, K. Fukami, T. Endo, and T. Takenawa, "A novel neural Wiskott-Aldrich syndrome protein (N-WASP) binding protein, WISH, induces Arp $2 / 3$ complex activation independent of Cdc42," The Journal of Cell Biology, vol. 152, no. 3, pp. 471-482, 2001.

[50] S. Lee, K. Lee, S. Hwang et al., "SPIN90/WISH interacts with PSD-95 and regulates dendritic spinogenesis via an $\mathrm{N}$ WASP-independent mechanism," The EMBO Journal, vol. 25, no. 20, pp. 4983-4995, 2006.

[51] L. A. Colgan and R. Yasuda, "Plasticity of dendritic spines: subcompartmentalization of signaling," Annual Review of Physiology, vol. 76, no. 1, pp. 365-385, 2014.

[52] Y. Kim, J. Y. Sung, I. Ceglia et al., "Phosphorylation of WAVE1 regulates actin polymerization and dendritic spine morphology," Nature, vol. 442, no. 7104, pp. 814-817, 2006. 